\title{
OUTPUT REgULATION OF SPROTT-F CHAOTIC SYSTEM BY STATE FEEDBACK CONTROL
}

\author{
Sundarapandian Vaidyanathan ${ }^{1}$ \\ ${ }^{1}$ Research and Development Centre, Vel Tech Dr. RR \& Dr. SR Technical University \\ Avadi, Chennai-600 062, Tamil Nadu, INDIA \\ sundarvtuegmail. com
}

\begin{abstract}
This paper solves the output regulation problem of Sprott-F chaotic system, which is one of the classical chaotic systems discovered by J.C. Sprott (1994). Explicitly, for the constant tracking problem, new state feedback control laws have been derived for regulating the output of the Sprott-F chaotic system. Our controller design has been carried out using the regulator equations of C.I. Byrnes and A. Isidori (1990). The output regulation of the Sprott-F chaotic system has important applications in many areas of Science and Engineering. Numerical simulations are shown to illustrate the effectiveness of the control schemes proposed in this paper for the output regulation of the Sprott-F chaotic system.
\end{abstract}

\section{KEYWORDS}

Chaos; Feedback Control; Sprott-F System; Nonlinear Control Systems; Output Regulation.

\section{INTRODUCTION}

In control systems literature, stabilization, control and output regulation are some important problems with applications in Science and Engineering. Basically, the output regulation problem is to control a fixed linear or nonlinear plant so that the output of the plant tracks reference signals produced by some external generator (the exosystem). For linear control systems, the output regulation problem has been solved by Francis and Wonham ([1], 1975). For nonlinear control systems, the output regulation problem was solved by Byrnes and Isidori ([2], 1990) generalizing the internal model principle obtained by Francis and Wonham [1]. Using Centre Manifold Theory [3], Byrnes and Isidori derived regulator equations, which characterize the solution of the output regulation problem of nonlinear control systems satisfying some stability assumptions.

The output regulation problem for nonlinear control systems has been studied extensively by various scholars in the last two decades [4-14]. In [4], Mahmoud and Khalil obtained results on the asymptotic regulation of minimum phase nonlinear systems using output feedback. In [5], Fridman solved the output regulation problem for nonlinear control systems with delay using centre manifold theory. In [6-7], Chen and Huang obtained results on the robust output regulation for output feedback systems with nonlinear exosystems. In [8], Liu and Huang obtained results on the global robust output regulation problem for lower triangular nonlinear systems with unknown control direction.

In [9], Immonen obtained results on the practical output regulation for bounded linear infinitedimensional state space systems. In [10], Pavlov, Van de Wouw and Nijmeijer obtained results 
on the global nonlinear output regulation using convergence-based controller design. In [11], Xi and Dong obtained results on the global adaptive output regulation of a class of nonlinear systems with nonlinear exosystems. In [12-14], Serrani, Isidori and Marconi obtained results on the semiglobal and global output regulation problem for minimum-phase nonlinear systems.

In this paper, we solve the output regulation problem for the Sprott-F chaotic system ([15], 1994). We find state feedback control laws solving the constant regulation problem of the Sprott-F chaotic system using the regulator equations of Byrnes and Isidori (1990). The Sprott-F chaotic system is a classical three-dimensional chaotic system discovered by J.C. Sprott (1994). It has important applications in Science and Engineering.

This paper is organized as follows. In Section 2, we provide a review the problem statement of output regulation problem for nonlinear control systems and the regulator equations of Byrnes and Isidori [2], which provide a solution to the output regulation problem under some stability assumptions. In Section 3, we present the main results of this paper, namely, the solution of the output regulation problem for the Sprott-F chaotic system for the important case of constant reference signals (set-point signals). In Section 4, we describe the numerical simulations for the state feedback controllers solving the output regulation problem for the Sprott-F chaotic system. In Section 5, we summarize the main results obtained in this paper.

\section{Review of the Output Regulation Problem For Nonlinear CONTROL SYSTEMS}

In this section, we consider a multi-variable nonlinear control system described by

$$
\begin{aligned}
& \dot{x}=f(x)+g(x) u+p(x) \omega \\
& \dot{\omega}=s(\omega) \\
& e=h(x)-q(\omega)
\end{aligned}
$$

Here, the differential equation (1a) describes the plant dynamics with state $x$ defined in a neighbourhood $X$ of the origin of $R^{n}$ and the input $u$ takes values in $R^{m}$ subject to te effect of a disturbance represented by the vector field $p(x) \omega$. The differential equation (1b) describes an autonomous system, known as the exosystem, defined in a neighbourhood $W$ of the origin of $R^{k}$, which models the class of disturbance and reference signals taken into consideration. The equation (2) defines the error between the actual plant output $h(x) \in R^{p}$ and a reference signal $q(\omega)$, which models the class of disturbance and reference signals taken into consideration.

We also assume that all the constituent mappings o the system (1) and the error equation (2), namely, $f, g, p, s, h$ and $q$ are continuously differentiable mappings vanishing at the origin, i.e.

$$
f(0)=0, g(0)=0, p(0)=0, s(0)=0, h(0)=0 \text { and } q(0)=0 .
$$

Thus, for $u=0$, the composite system (1) has an equilibrium $(x, \omega)=(0,0)$ with zero error $(2)$.

A state feedback controller for the composite system (1) has the form

$$
u=\rho(x, \omega)
$$


where $\rho$ is a continuously differentiable mapping defined on $X \times W$ such that $\rho(0,0)=0$.

Upon substitution of the feedback control law (3) into (1), we get the closed-loop system

$$
\begin{aligned}
& \dot{x}=f(x)+g(x) \rho(x, \omega)+p(x) \omega \\
& \dot{\omega}=s(\omega)
\end{aligned}
$$

The purpose of designing the state feedback controller (3) is to achieve both internal stability and output regulation of the given nonlinear control system (1). Formally, we can summarize these requirements as follows.

\section{State Feedback Regulator Problem [2]:}

Find, if possible, a state feedback control law $u=\rho(x, \omega)$ such that the following conditions are satisfied.

(OR1) [Internal Stability] The equilibrium $x=0$ of the dynamics

$$
\dot{x}=f(x)+g(x) \rho(x, 0)
$$

is locally exponentially stable.

(OR2) [Output Regulation] There exists a neighbourhood $U \subset X \times W$ of $(x, \omega)=(0,0)$ such that for each initial condition $(x(0), \omega(0)) \in U$, the solution $(x(t), \omega(t))$ of the closed-loop system (4) satisfies

$$
\lim _{t \rightarrow \infty}[h(x(t))-q(\omega(t))]=0 .
$$

Byrnes and Isidori [2] solved the output regulation problem stated above under the following two assumptions.

(H1) The exosystem dynamics $\dot{\omega}=s(\omega)$ is neutrally stable at $\omega=0$, i.e. the exosystem is Lyapunov stable in both forward and backward time at $\omega=0$.

(H2) The pair $(f(x), g(x))$ has a stabilizable linear approximation at $x=0$, i.e. if

$$
A=\left[\frac{\partial f}{\partial x}\right]_{x=0} \text { and } B=\left[\frac{\partial g}{\partial x}\right]_{x=0}
$$

then $(A, B)$ is stabilizable.

Next, we recall the solution of the output regulation problem derived by Byrnes and Isidori [2].

Theorem 1. [2] Under the hypotheses (H1) and (H2), the state feedback regulator problem is solvable if and only if there exist continuously differentiable mappings $x=\pi(\omega)$ with $\pi(0)=0$ 
and $u=\varphi(\omega)$ with $\varphi(0)=0$, both defined in a neighbourhood of $W^{0} \subset W$ of $\omega=0$ such that the following equations (called the regulator equations) are satisfied:

(1) $\frac{\partial \pi}{\partial \omega} s(\omega)=f(\pi(\omega))+g(\pi(\omega)) \varphi(\omega)+p(\pi(\omega)) \omega$

(2) $h(\pi(\omega))-q(\omega)=0$

When the regulator equations (1) and (2) are satisfied, a control law solving the state feedback regulator problem is given by

$$
u=\varphi(\omega)+K[x-\pi(\omega)]
$$

where $K$ is any gain matrix such that $A+B K$ is Hurwitz.

\section{OUtPut Regulation OF ThE SPRotT-F ChaOtic System}

In this section, we solve the output regulation problem for the Sprott-F system ([15], 1994), which is one of the paradigms of the 3-dimensional chaotic systems described by the dynamics

$$
\begin{aligned}
& \dot{x}_{1}=x_{2}+x_{3} \\
& \dot{x}_{2}=-x_{1}+a x_{2} \\
& \dot{x}_{3}=x_{1}^{2}-b x_{3}+u
\end{aligned}
$$

where $x_{1}, x_{2}, x_{3}$ are the states of the system, $a, b$ are positive, constant parameters of the system and $u$ is the scalar control. 


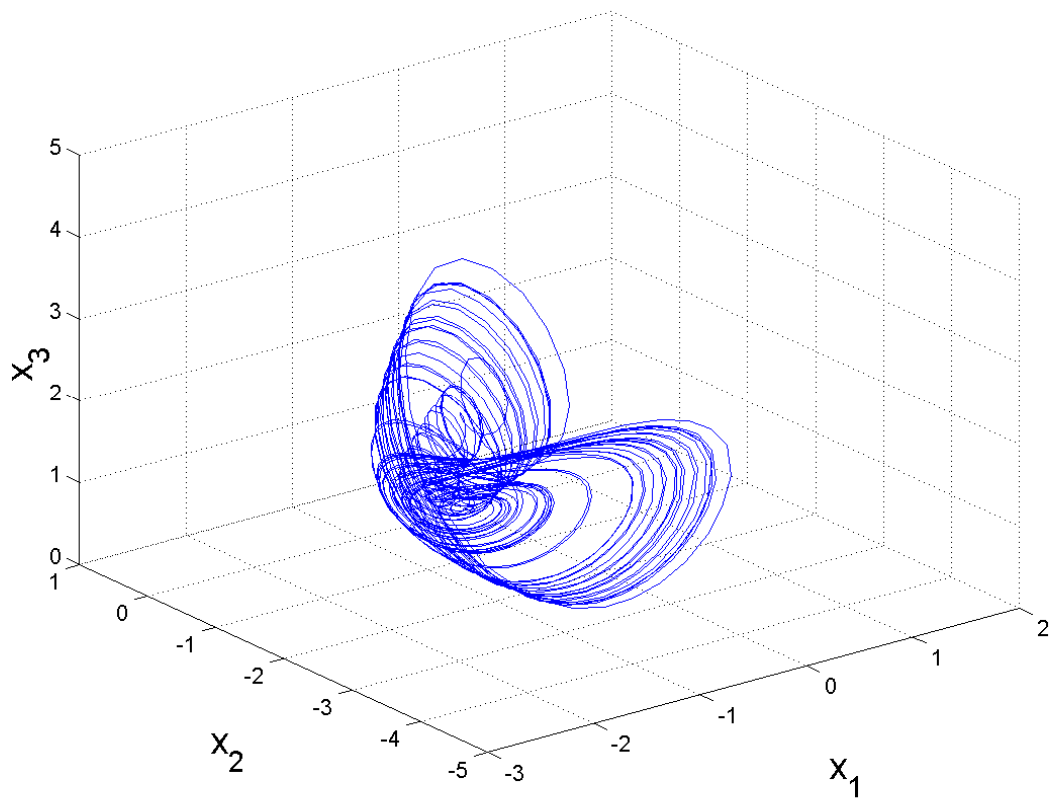

Figure 1. Strange Attractor of the Sprott-F Chaotic System

J.C. Sprott ([15], 1994) showed that the system (5) has chaotic behaviour when $a=0.5, b=1$ and $u=0$. The strange attractor of the Sprott-F chaotic system is illustrated in Figure 1 .

In this paper, we consider the output regulation problem for the tracking of constant reference signals (set-point signals).

In this case, the exosystem is given by the scalar dynamics

$$
\dot{\omega}=0
$$

We note that the assumption (H1) of Theorem 1 holds trivially.

Linearizing the dynamics of the Sprott-F chaotic system (5) at $x=0$, we obtain

$$
A=\left[\begin{array}{ccc}
0 & 1 & 1 \\
-1 & a & 0 \\
0 & 0 & -b
\end{array}\right] \text { and } B=\left[\begin{array}{l}
0 \\
0 \\
1
\end{array}\right]
$$

Using Kalman's rank test for controllability ([16], p738), it can be easily seen that the pair $(A, B)$ is completely controllable.

Thus, it follows that the pair $(A, B)$ is stabilizable.

Thus, the assumption (H2) of Theorem 1 also holds.

Hence, Theorem 1 can be applied to solve the constant regulation problem for the Sprott-I chaotic system (5). 


\subsection{The Constant Tracking Problem for $x_{1}$}

Here, the tracking problem for the Sprott-I chaotic system (5) is given by

$$
\begin{aligned}
& \dot{x}_{1}=x_{2}+x_{3} \\
& \dot{x}_{2}=-x_{1}+a x_{2} \\
& \dot{x}_{3}=x_{1}^{2}-b x_{3}+u \\
& e=x_{1}-\omega
\end{aligned}
$$

By Theorem 1, the regulator equations of the system (8) are obtained as

$$
\begin{array}{r}
\pi_{2}(\omega)+\pi_{3}(\omega)=0 \\
-\pi_{1}(\omega)+a \pi_{2}(\omega)=0 \\
\pi_{1}^{2}(\omega)-b \pi_{3}(\omega)+\varphi(\omega)=0 \\
\pi_{1}(\omega)-\omega=0
\end{array}
$$

Solving the regulator equations (9) for the system (8), we obtain the unique solution as

$$
\pi_{1}(\omega)=\omega, \quad \pi_{2}(\omega)=\frac{\omega}{a}, \quad \pi_{3}(\omega)=-\frac{\omega}{a} \text { and } \varphi(\omega)=-\omega^{2}-\frac{b \omega}{a}
$$

Using Theorem 1 and the solution (10) of the regulator equations for the system (8), we obtain the following result which provides a solution of the output regulation problem for (8).

Theorem 2. A state feedback control law solving the output regulation problem for the Sprott-F chaotic system (8) is given by

$$
u=\varphi(\omega)+K[x-\pi(\omega)]
$$

where $\varphi(\omega), \pi(\omega)$ are defined as in (10) and $K$ is chosen so that $A+B K$ is Hurwitz.

\subsection{The constant Tracking Problem for $x_{2}$}

Here, the tracking problem for the Sprott-I chaotic system (5) is given by

$$
\begin{aligned}
& \dot{x}_{1}=x_{2}+x_{3} \\
& \dot{x}_{2}=-x_{1}+a x_{2} \\
& \dot{x}_{3}=x_{1}^{2}-b x_{3}+u \\
& e=x_{2}-\omega
\end{aligned}
$$

By Theorem 1, the regulator equations of the system (12) are obtained as 
International Journal of Control Theory and Computer Modelling (IJCTCM) Vol.2, No.2, March 2012

$$
\begin{array}{r}
\pi_{2}(\omega)+\pi_{3}(\omega)=0 \\
-\pi_{1}(\omega)+a \pi_{2}(\omega)=0 \\
\pi_{1}^{2}(\omega)-b \pi_{3}(\omega)+\varphi(\omega)=0 \\
\pi_{2}(\omega)-\omega=0
\end{array}
$$

Solving the regulator equations (13) for the system (12), we obtain the unique solution as

$$
\pi_{1}(\omega)=a \omega, \quad \pi_{2}(\omega)=\omega, \quad \pi_{3}(\omega)=-\omega \text { and } \varphi(\omega)=-a^{2} \omega^{2}-b \omega
$$

Using Theorem 1 and the solution (14) of the regulator equations for the system (13), we obtain the following result which provides a solution of the output regulation problem for (13).

Theorem 3. A state feedback control law solving the output regulation problem for the Sprott-F chaotic system (12) is given by

$$
u=\varphi(\omega)+K[x-\pi(\omega)]
$$

where $\varphi(\omega), \pi(\omega)$ are defined as in (14) and $K$ is chosen so that $A+B K$ is Hurwitz.

\subsection{The Constant Tracking Problem for $x_{3}$}

Here, the tracking problem for the Sprott-I chaotic system (5) is given by

$$
\begin{aligned}
& \dot{x}_{1}=x_{2}+x_{3} \\
& \dot{x}_{2}=-x_{1}+a x_{2} \\
& \dot{x}_{3}=x_{1}^{2}-b x_{3}+u \\
& e=x_{3}-\omega
\end{aligned}
$$

By Theorem 1, the regulator equations of the system (16) are obtained as

$$
\begin{aligned}
\pi_{2}(\omega)+\pi_{3}(\omega) & =0 \\
-\pi_{1}(\omega)+a \pi_{2}(\omega) & =0 \\
\pi_{1}^{2}(\omega)-b \pi_{3}(\omega)+\varphi(\omega) & =0 \\
\pi_{3}(\omega)-\omega & =0
\end{aligned}
$$

Solving the regulator equations (17) for the system (16), we obtain the unique solution as

$$
\pi_{1}(\omega)=-a \omega, \quad \pi_{2}(\omega)=-\omega, \quad \pi_{3}(\omega)=\omega \text { and } \varphi(\omega)=-a^{2} \omega^{2}+b \omega
$$

Using Theorem 1 and the solution (18) of the regulator equations for the system (16), we obtain the following result which provides a solution of the output regulation problem for (16).

Theorem 4. A state feedback control law solving the output regulation problem for the Sprott-F chaotic system (16) is given by

$$
u=\varphi(\omega)+K[x-\pi(\omega)]
$$


International Journal of Control Theory and Computer Modelling (IJCTCM) Vol.2, No.2, March 2012

where $\varphi(\omega), \pi(\omega)$ are defined as in (16) and $K$ is chosen so that $A+B K$ is Hurwitz.

\section{NUMERICAL SimUlationS}

For simulation, the parameters are chosen as the chaotic case of the Sprott-F system, viz. $a=0.5$ and $b=1$.

For achieving internal stability of the state feedback regulator problem, a feedback gain matrix $K$ must be chosen so that $A+B K$ is Hurwitz.

Suppose we wish to choose a gain matrix $K$ such that the closed-loop system matrix $A+B K$ has stable eigenvalues $\{-5,-5,-5\}$.

Since $(A, B)$ is controllable, we obtain the gain matrix $K$ by Ackermann's formula (MATLAB) as

$$
K=\left[\begin{array}{lll}
-81.75 & 150.375 & -14.5
\end{array}\right]
$$

For the numerical simulations, the fourth order Runge-Kutta method with step-size $h=10^{-8}$ is deployed to solve the systems of differential equations using MATLAB.

\subsection{Constant Tracking Problem for $x_{1}$}

Here, the initial conditions are taken as

$$
x_{1}(0)=18, x_{2}(0)=-4, x_{3}(0)=10 \text { and } \omega=2
$$

The simulation graph is depicted in Figure 2 from which it is clear that the state trajectory $x_{1}(t)$ tracks the constant reference signal $\omega=2$ in 3 seconds. 
International Journal of Control Theory and Computer Modelling (IJCTCM) Vol.2, No.2, March 2012

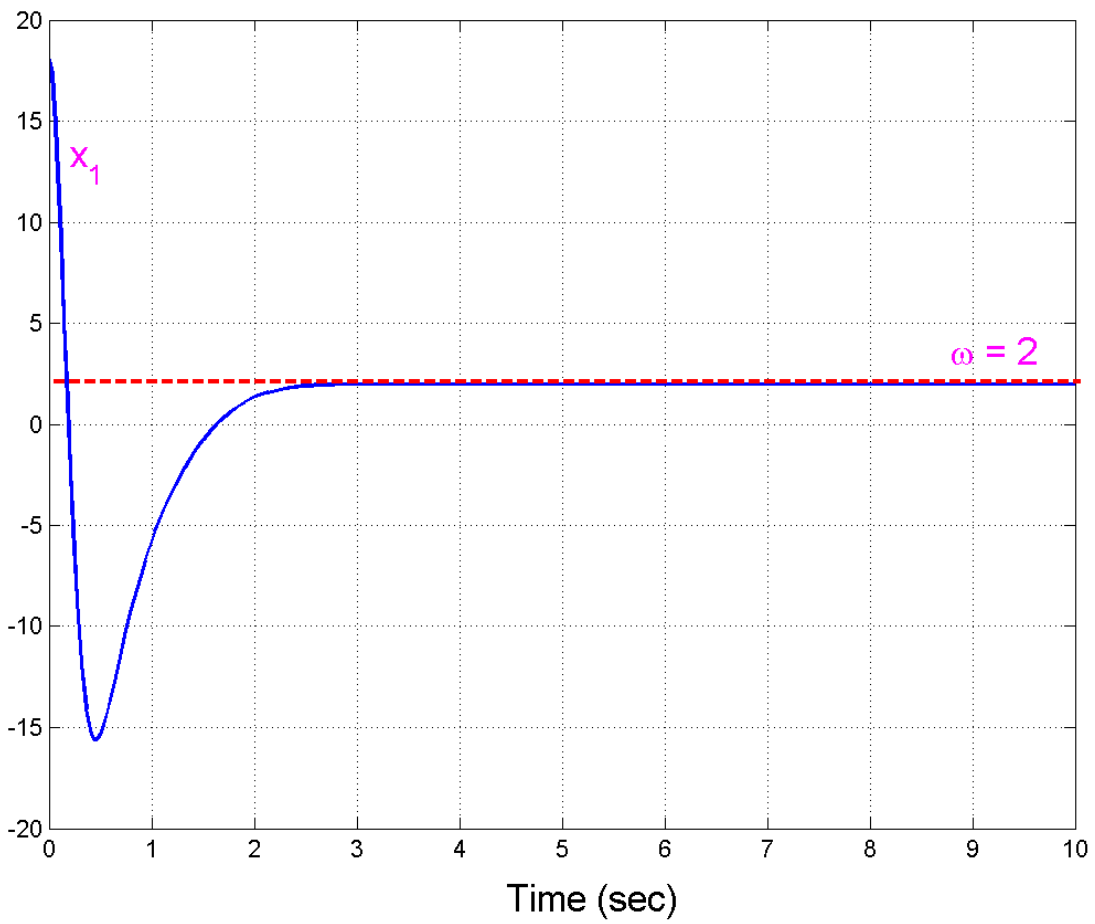

Figure 2. Constant Tracking Problem for $x_{1}$

\subsection{Constant Tracking Problem for $x_{2}$}

Here, the initial conditions are taken as

$$
x_{1}(0)=5, \quad x_{2}(0)=14, x_{3}(0)=-10 \quad \text { and } \omega=2
$$

The simulation graph is depicted in Figure 3 from which it is clear that the state trajectory $x_{2}(t)$ tracks the constant reference signal $\omega=2$ in 3 seconds. 
International Journal of Control Theory and Computer Modelling (IJCTCM) Vol.2, No.2, March 2012

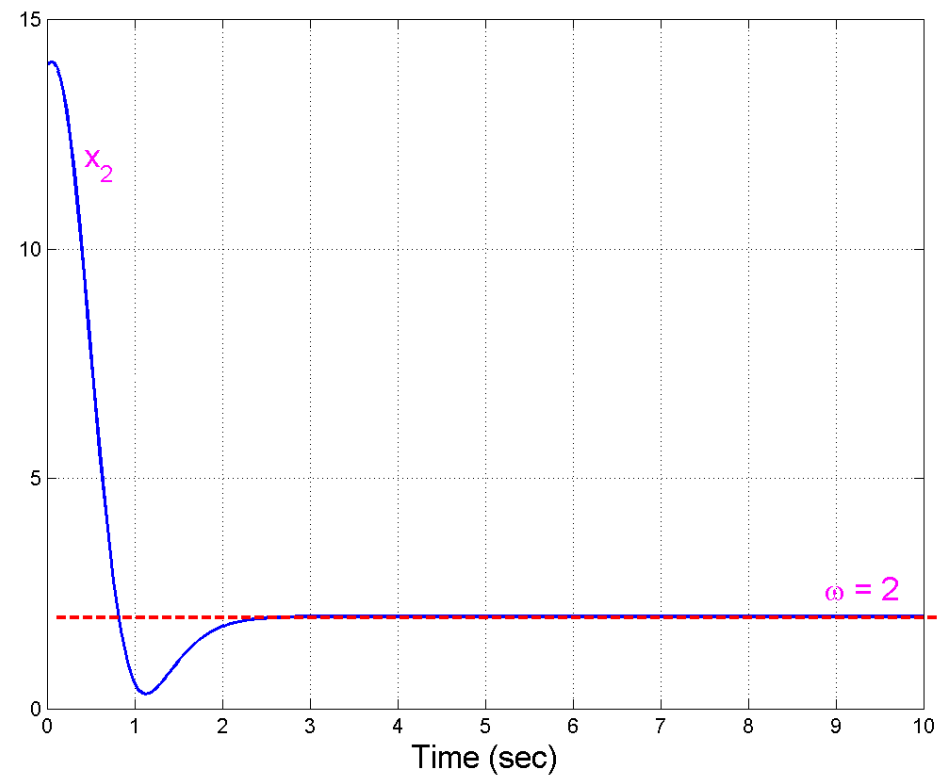

Figure 3. Constant Tracking Problem for $x_{2}$

\subsection{Constant Tracking Problem for $x_{3}$}

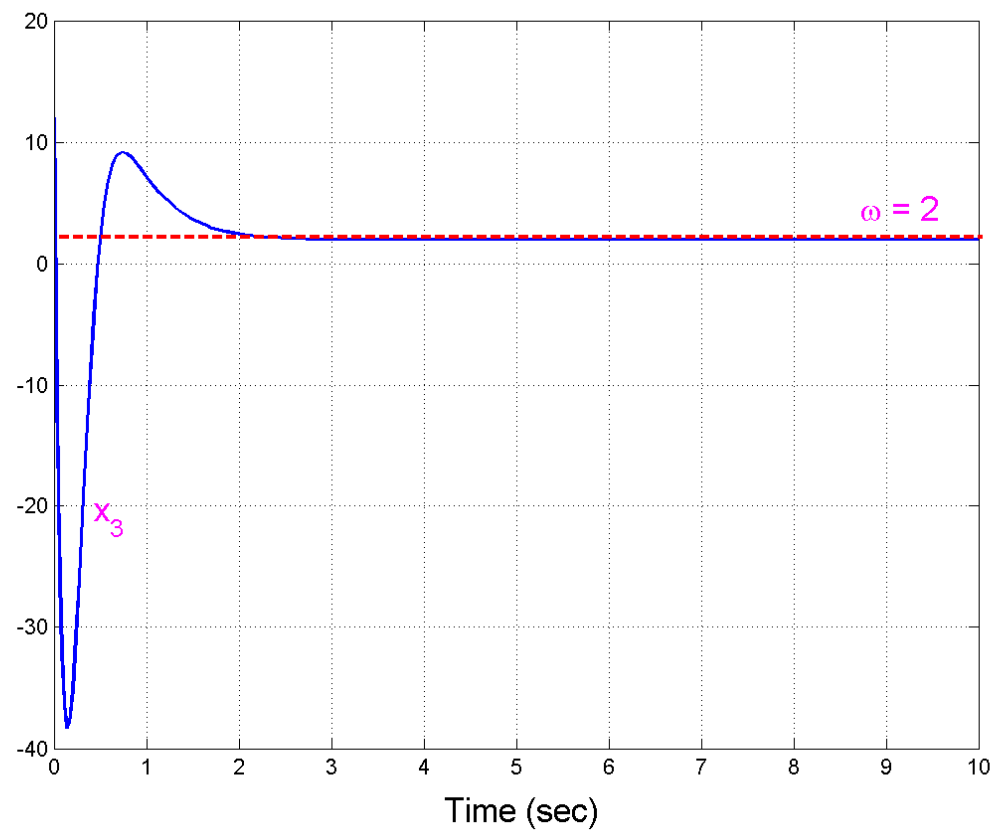

Figure 4. Constant Tracking Problem for $x_{3}$ 
Here, the initial conditions are taken as

$$
x_{1}(0)=7, x_{2}(0)=-3, x_{3}(0)=12 \text { and } \omega=2 \text {. }
$$

The simulation graph is depicted in Figure 4 from which it is clear that the state trajectory $x_{3}(t)$ tracks the constant reference signal $\omega=2$ in 3 seconds.

\section{CONClusions}

In this paper, the output regulation problem for the Sprott-F chaotic system (1994) has been studied in detail and a complete solution for the output regulation problem for the Sprott-F chaotic system has been derived for the tracking of constant reference signals (set-point signals). The state feedback control laws achieving output regulation proposed in this paper were derived using the regulator equations of Byrnes and Isidori (1990). Numerical simulation results were presented in detail to illustrate the effectiveness of the proposed control schemes for the output regulation problem of Sprott-F chaotic system to track constant reference signals.

\section{REFERENCES}

[1] Francis, B.A. \& Wonham, W.M. (1975) "The internal model principle for linear multivariable regulators", J. Applied Math. Optimization, Vol. 2, pp 170-194.

[2] Byrnes, C.I. \& Isidori, A. (1990) "Output regulation of nonlinear systems", IEEE Trans. Automatic Control, Vol. 35, pp 131-140.

[3] Carr, J. (1981) Applications of Centre Manifold Theory, Springer Verlag, New York.

[4] Mahmoud, N.A. \& Khalil, H.K. (1996) "Asymptotic regulation of minimum phase nonlinear systems using output feedback”, IEEE Trans. Automat. Control, Vol. 41, pp 1402-1412.

[5] Fridman, E. (2003) "Output regulation of nonlinear control systems with delay", Systems \& Control Lett., Vol. 50, pp 81-93.

[6] Chen, Z. \& Huang, J. (2005) "Robust output regulation with nonlinear exosystems", Automatica, Vol. 41, pp 1447-1454.

[7] Chen, Z. \& Huang, J. (2005) “Global robust output regulation for output feedback systems", IEEE Trans. Automat. Control, Vol. 50, pp 117-121.

[8] Liu, L. \& Huang, J. (2008) "Global robust output regulation of lower triangular systems with unknown control direction”, Automatica, Vol. 44, pp. 1278-1284.

[9] Immonen, E. (2007) "Practical output regulation for bounded linear infinite-dimensional state space systems”, Automatica, Vol. 43, pp 786-794.

[10] Pavlov, A., Van de Wouw, N. \& Nijmeijer, H. (2007) "Global nonlinear output regulation: convergence based controller design", Automatica, Vol. 43, pp 456-463.

[11] Xi, Z. \& Ding, Z. (2007) "Global robust output regulation of a class of nonlinear systems with nonlinear exosystems”, Automatica, Vol. 43, pp 143-149.

[12] Serrani, A. \& Isidori, A. (2000) "Global robust output regulation for a class of nonlinear systems", Systems \& Control Letters, Vol. 39, pp 133-139. 
International Journal of Control Theory and Computer Modelling (IJCTCM) Vol.2, No.2, March 2012

[13] Serrani, A., Isidori, A. \& Marconi, L. (2000) "Semiglobal output regulation for minimum phase systems", Internat. J. Robust Nonlinear Control, Vol. 10, pp 379-396.

[14] Marconi, L., Isidori, A. \& Serrani, A. (2004) "Non-resonance conditions for uniform observability in the problem of nonlinear output regulation", Systems \& Control Letters, Vol. 53, pp 281-298.

[15] Sprott, J.C. (1994) "Some simple chaotic flows," Physical Review E, Vol. 50, No. 2, pp. 647-650.

[16] Ogata, K. (1997) Modern Control Engineering, Prentice Hall, New Jersey, U.S.A.

[17] Sundarapandian, V. (2011) "Output regulation of Shimizu-Morioka chaotic system by state feedback control”, International J. Advanced Information Technology, Vol. 1, No. 2, pp. 1-10.

\section{Author}

Dr. V. Sundarapandian obtained his Doctor of Science degree in Electrical and Systems Engineering from Washington University, St. Louis, USA in May 1996. He is a Professor at the $\mathrm{R} \& \mathrm{D}$ Centre at Vel Tech Dr. RR \& Dr. SR Technical University, Chennai, Tamil Nadu, India. He has published over 240 refereed international publications. He has published over 160 papers in National and International Conferences. He is the Editor-in-Chief of the AIRCC Journals International Journal of Instrumentation and Control Systems, International Journal of Control Systems and Computer Modelling, and International Journal of Information Technology, Control and Automation. His research interests are Linear and Nonlinear Control Systems, Chaos Theory and Control, Soft Computing,

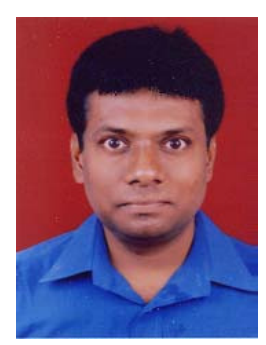
Optimal Control, Operations Research, Mathematical Modelling and Scientific Computing. He has delivered several Key Note Lectures on Control Systems, Chaos Theory, Scientific Computing, Mathematical Modelling, MATLAB and SCILAB. 